\title{
ARTICLE
}

Received $1^{\text {st }}$ September 2020 Accepted $13^{\text {rd }}$ October 2020 DOI: $\underline{10.1039 / D O S M 01581 D}$

\section{Membrane reinforcement in Giant Hybrid Polymer Lipid Vesicles achieved by controlling the polymer architecture}

\author{
Martin Fauquignon, ${ }^{a}$ Emmanuel Ibarboure, ${ }^{a}$ Jean-François Le Meins ${ }^{a} *$ \\ Physical properties of membranes of hybrid polymer lipid vesicles are so far relatively unknown. Since their discovery a \\ decade ago, many studies have aimed to show their high potential in many fields of application, but so far, few systematic \\ studies have been carried out to decipher the relationship between the molecular characteristic of the components (molar \\ mass, chemical nature, architecture of the copolymer), the membrane structuration and its properties. In this work, we \\ study the association of 1-palmitoyl-2-oleoyl-sn-glycero-3-phosphocholine (POPC) and poly(dimethylsiloxane)- $b$ - \\ poly(ethylene oxide) (PDMS- $b$-PEO) diblock copolymers of different molar masses in giant hybrid vesicle and establish a \\ complete phase diagram of the membrane structuration. We also measure the mechanical properties of the GHUV \\ through micropipette aspiration at different lipid/polymer compositions. Thanks to a previous work using triblock PEO- $b$ - \\ PDMS- $b$-PEO copolymers, we were able to reveal the effect of the architecture of block copolymer on membrane \\ structuration and properties. Besides, the association of diblock copolymers PDMS- $b$-PEO with POPC lead to the formation \\ of hybrid vesicle with unprecedented membrane toughness.
}

\section{Introduction}

Hybrid vesicles are the subject of a growing number of studies by different scientific communities (chemists, physicochemists, biophysicists, biologists, pharmacists) these last years. This is due to the fact that these structures could synergistically combine the advantages of each component (permeability and biofunctionality of lipid bilayers, chemical versatility and mechanical stability of polymer membranes). This is of great interest in applications such as controlled and targeted drug delivery, development of artificial cells or bioinspired micro/nanoreactors. Although the field of research is still in its infancy age, several reviews have been written on the subject ${ }^{1-4}$ and a special issue in the journal polymers is focused on these systems.

https://www.mdpi.com/journal/polymers/special issues/hybri d polymer lipid.

So far many studies have sought to highlight their potential interest for different pharmaceutical, biological or biochemical applications such as bioreactor, ${ }^{5-12}$ drug cargo ${ }^{13-19}$ or molecular recognition tool. ${ }^{20}$ A relatively few number of studies have focused on the self-assembly and the relationship between membrane structuration and properties of these hybrid systems. ${ }^{21}{ }^{22,}{ }^{23}$ Molecular and macroscopic parameters which

\footnotetext{
aniversité de Bordeaux, CNRS, Bordeaux INP, LCPO, UMR 5629, F-33600, Pessac, France.

* corresponding author lemeins@enscbp.fr

Electronic Supplementary Information (ESI) available: Stress strain curves, analysis of influence of molecular probes on mechanical properties. See DOI: $10.1039 / D 0 S M 01581 \mathrm{D}$ or at the end of this author manuscript version
}

govern the membrane structuration toward homogenous mixing of lipid and polymers or to phase separation leading to the presence of micron size or nanoscale domain are partially known. It is however of great importance to understand and ultimately control their properties in order to obtain optimized systems for future biomedical applications.

At the micrometric scale, a homogeneous distribution of the components seems to be favoured if the lipid phase is in a fluid state and at a relatively low fraction. ${ }^{23-26}$ Above a given lipid fraction, not yet rationalized in the literature, micrometric domains start to appear and eventually evolve towards budding and fission phenomena depending on the line tension at the lipid domains boundaries. This parameter is partially modulated by the hydrophobic length mismatch at the polymer/lipid interface and consequently by the molar mass of the hydrophobic part of the copolymers. Although this parameter plays a role in the structuration of hybrid membranes, it has never been quantified for these systems at this time.

Regarding their membrane properties, most of the studies focused on the membrane permeability. They have been evaluated so far essentially trough drug release experiments. ${ }^{15,10,16,27,28}$ However, it is relatively hard to extract general conclusion on the diffusivity of a molecule through a hybrid membrane as the systems and loaded molecules were variable and membrane structure were not resolved. Information about thermomechanical properties are also relatively scarce. Studies on PBD- $b$-PEO/POPC ${ }^{25}$ and PEO$b$-PDMS- $b$-PEO/POPC ${ }^{29}$ hybrid giant vesicle realized through 
micropipettes techniques have shown a modulation of area expansion modulus by the membrane composition. However, a dramatical decrease of the membrane toughness in the case or triblock PEO- $b$-PDMS- $b$-PEO copolymer was observed. Another study on PDMS-g-PEO/DPPC giant hybrid unilamellar vesicle, mentions that area expansion modulus of hybrid membrane was almost unchanged upon addition of DPPC, compared to the polymer membrane. ${ }^{30}$ In another study, membrane modulus of DPPC vesicle obtained by measuring shrinking of GUV during osmotic shock experiments, was increased by lateral co-assembly of PEO- $b$-PDMS- $b$-PEO copolymers or PEO- $b$-PCL- $b$-PEO. ${ }^{31}$ Again, there is no clear trend regarding the influence of the polymer/lipid composition and membrane structuration on the mechanical properties of the resulting hybrid membrane.

In this work we propose a complete investigation of the membrane mechanical properties of GHUV obtained with diblock copolymers PDMS- $b$-PEO and POPC and compared the results obtained in a previous study with triblock copolymers PEO- $b$-PDMS- $b$-PEO. ${ }^{29}$ We aim at conclude on the effect of the architecture of the copolymer on the mechanical properties of the resulting hybrid vesicles. We focused on a range of lipid composition where homogenous membranes were obtained, at least at the micrometric scale.

\section{Materials and Methods}

\section{Materials.}

1-palmitoyl-2-oleoyl-sn-glycero-3-phosphocholine (POPC), 1,2dioleoyl-sn-glycero-3-phosphoethanol-amine- $\mathrm{N}$-(lissamine rhodamine B sulfonyl) (PE-rhodamine), sucrose, glucose, bovine serum albumin and all organic solvents were purchased from Sigma Aldrich and used without further purification. The different PDMS- $b$-PEO copolymers were synthesized according to previously described protocol. $^{32}$ A brief recall of the synthesis procedure is available in ESI. The molecular characteristics of the copolymers are illustrated in Table 1.

\begin{tabular}{|c|c|c|c|c|}
\hline Compound & Abbrev. & $\begin{array}{c}\text { Molar } \\
\text { mass }^{\text {a }} \\
(\text { g.mol }\end{array}$ & $\begin{array}{c}\text { Hydrophobic } \\
\text { molar mass } \\
\left(\text { a } \text { mol }^{-1} \text { ) }\right.\end{array}$ & $\begin{array}{c}\text { Membrane } \\
\text { thickness } \\
(\mathrm{nm})\end{array}$ \\
\hline $\begin{array}{l}\text { 1-palmitoyl-2-oleoyl- } \\
\text { sn-glycero-3- } \\
\text { phosphocholine }\end{array}$ & POPC & $\begin{array}{c}760 \\
-\end{array}$ & - & $4.7 \pm 0.9$ \\
\hline $\mathrm{PDMS}_{23}-b-\mathrm{PEO}_{13}{ }^{32}$ & $\mathrm{Si}_{23} \mathrm{EO}_{13}$ & $\begin{array}{c}2500 \\
1.15\end{array}$ & 1700 & $6.9 \pm 1.0$ \\
\hline $\mathrm{PDMS}_{27}-b-\mathrm{PEO}_{17}{ }^{32}$ & $\mathrm{Si}_{27} \mathrm{EO}_{17}$ & $\begin{array}{c}2900 \\
1.11\end{array}$ & 2000 & $8.4 \pm 1.1$ \\
\hline $\mathrm{PDMS}_{36}-b-\mathrm{PEO}_{23}{ }^{32}$ & $\mathrm{Si}_{36} \mathrm{EO}_{23}$ & $\begin{array}{l}4000 \\
1.04\end{array}$ & 2700 & $9.9 \pm 1.6$ \\
\hline $\begin{array}{c}\mathrm{PEO}_{8}-b-\mathrm{PDMS}_{22}-b- \\
\mathrm{PEO}_{8}^{23,29}\end{array}$ & $\mathrm{EO}_{8} \mathrm{Si}_{22} \mathrm{EO}_{8}$ & $\begin{array}{l}2700 \\
1.18\end{array}$ & 1600 & $5.4 \pm 0.4$ \\
\hline $\begin{array}{c}\mathrm{PEO}_{12}-b-\mathrm{PDMS}_{43}-b- \\
\mathrm{PEO}_{12}{ }^{23,29}\end{array}$ & $\mathrm{EO}_{12} \mathrm{Si}_{43} \mathrm{EO}_{12}$ & $\begin{array}{l}5000 \\
1.28\end{array}$ & 3200 & $8.8 \pm 0.5$ \\
\hline
\end{tabular}

Table 1. Molecular characteristics of the copolymers and the phospholipid used in this work and membrane thickness of the vesicle obtained. Molecular characteristic of triblock copolymer used in previous studies ${ }^{23,29}$ are recalled. ${ }^{\text {a }}$ Molar mass determined by ${ }^{1} \mathrm{H}$ NMR. ${ }^{b}$ Membrane thickness determined by SANS in ref $^{32,33}$.

\section{GHUV Preparation}

All Giant Unilamellar Vesicles in this study were prepared by the same process based on the electroformation method reported by Angelova and Dimitrov ${ }^{34}$ at room temperature. The probes were used at $0.2 \mathrm{wt} . \%$ for PE-rhodamine and 1.5 wt.\% for PDMS-NBD. Briefly, mixtures of the appropriate lipids, polymers, and probes were prepared in chloroform at a total concentration of $1 \mathrm{mg} \cdot \mathrm{ml}^{-1}$. About $50 \mu \mathrm{l}$ of this solution was spread slowly on the conductive faces of two indium tin oxide coated glass slides (ITO, 30-60 $\Omega$ /sq from Sigma Aldrich). The slides were sealed on both sides of a rubber spacer using grease to form a chamber and dried under vacuum for $3 \mathrm{~h}$. The slides were connected to $A C$ voltage generator using electric wires and a typical sinusoidal tension $(2 \mathrm{~V}, 10 \mathrm{~Hz})$ was applied and the chamber was filled with a $100 \mathrm{mM}$ sucrose solution. The vesicles were collected after 75 minutes and kept in the dark at room temperature.

\section{Confocal imaging}

All images were acquired on a Leica TCS SP5 (Leica Microsystems CMS GmbH, Mannheim, Germany) inverted confocal microscope (DMI6000) equipped with a HCX PL APO x63 NA 1.4 oil immersion objective (Zeiss). A 50- $\mu \mathrm{L}$ aliquot of the GUV suspension was added into an eight-well $\mu$-Slide (Ibidi, Martinsried, Germany) containing $150 \mu \mathrm{L}$ iso-osmolar (100 $\mathrm{mM}$ ) glucose solution. GUVs were allowed to sediment for 10 min before imaging. PDMS-NBD and rhodamine-PE were used as markers of the polymer and lipid phases respectively and were separately imaged using an argon laser line with an excitation/range of emission, respectively of $488 \mathrm{~nm} / 500-530$ $\mathrm{nm}$ and $514 \mathrm{~nm} / 600-700 \mathrm{~nm}$. Images were processed using Leica and ImageJ software.

\section{Micropipette aspiration experiment}

The micropipette aspiration technique was also carried out on a Leica TCS SP5 (Leica Microsystems CMS GmbH, Mannheim, Germany) inverted confocal microscope (DMI6000) equipped with a $x 63$ apochromatic water immersion objective with a NA of 1.2 (Zeiss, Jena Germany). Micropipettes were obtained by stretching Borosilicate capillaries ( $1 \mathrm{~mm} \mathrm{OD}, 0.58 \mathrm{~mm}$ ID) from WPI, using a pipette puller (Sutter Instrument P-97). The pulled pipettes were then forged to the desired diameter using a micro-forge Narishige MF-900. Micropipettes were coated with BSA to prevent vesicle adhesion. The vesicle tension was controlled using a home-made hydraulic watertight setup, and the micropipette was controlled using a micromanipulator (Eppendorf, Patchman NP2). 
The suction pressure exerted over the membrane can be calculated from:

$$
\Delta \mathrm{P}=\left(\mathrm{h}-\mathrm{h}_{0}\right) \rho \mathrm{g}
$$

where $\rho$ is the water density, $g$ is the gravitational acceleration, $h$ is the position of the water tank and $h_{0}$ is the initial position where the pressure is zero.

The membrane tension was calculated classically from the Laplace equation:

$$
\sigma=\frac{\Delta \mathrm{P}}{2} \frac{\mathrm{R}_{\mathrm{P}}}{\left(1-\frac{\mathrm{R}_{\mathrm{P}}}{\mathrm{R}_{\mathrm{V}}}\right)}
$$

where $R_{p}$ and $R_{v}$ are respectively the micropipette and vesicle radius

(outside the micropipette), and $\Delta P$ the suction pressure. The relative area change of the membrane $(\alpha)$ is defined as:

$$
\alpha=\frac{\mathrm{A}-\mathrm{A}_{0}}{\mathrm{~A}_{0}}
$$

$A_{0}$ being the membrane area of the vesicle at the lower suction pressure. $\alpha$ can be estimated from the increase in projection length $\Delta \mathrm{L}$ of the vesicle inside the capillary tip according to equation (4):

$$
\alpha=\frac{1}{2}\left(\frac{\mathrm{R}_{\mathrm{P}}}{\mathrm{R}_{\mathrm{V}}}\right)^{2}\left(1-\frac{\mathrm{R}_{\mathrm{P}}}{\mathrm{R}_{\mathrm{V}}}\right) \frac{\Delta \mathrm{L}}{\mathrm{R}_{\mathrm{P}}}
$$

The surface area strain can be linked to the membrane tension through the following equation:

$$
\alpha=\frac{\mathrm{kT}}{8 \pi \mathrm{K}_{\mathrm{b}}} \ln \left(1+\frac{\mathrm{A}_{0} \sigma}{24 \pi \mathrm{K}_{\mathrm{b}}}\right)+\frac{\sigma}{\mathrm{K}_{\mathrm{a}}}
$$

Where $\mathrm{k}$ is the Boltzmann constant, $\mathrm{T}$ the temperature and $\mathrm{K}_{\mathrm{b}}$ and $\mathrm{K}_{\mathrm{a}}$ respectively the bending and stretching modulus. According to previous work, at high tension the membrane undulations are completely suppressed and membrane area increases as the result of increased spacing between molecule, giving access to area compressibility modulus:

$$
\sigma=\mathrm{K}_{\mathrm{a}} \alpha\left(1-\frac{5}{4} \alpha\right)
$$

Each parameter measured in this work is taken from an average value of at least 15 vesicles unless specified. The vesicles were analyzed one hour after electroformation.

Detailed protocol ${ }^{35}$ and video demonstration how to set-up a micropipette aspiration experiment is also available at the following link:

https://www.jove.com/video/60199/obtention-giant-unilamellarhybrid-vesicles-electroformation

It has to be noted that micropipette experiments have been performed without fluorescent probes, as an effect have been detected on mechanical properties, as described in supporting information (Figure S1).

\section{Results}

\section{Membrane structuration}

The three diblock copolymers $\left(\mathrm{Si}_{23} \mathrm{EO}_{13}, \mathrm{Si}_{27} \mathrm{EO}_{17}\right.$ and $\left.\mathrm{Si}_{36} \mathrm{EO}_{23}\right)$ were tested for the formation of GHUV, using different proportions of POPC. The structuration of the GHUV's membrane were studied by confocal microscopy on a population large enough (between 200 and 800 objects counted per sample) to be representative. The proportion of each structuration was counted manually from images taken in different randomly selected areas of the sample. Depending on the copolymer used and the POPC fraction, we could observe different structuration (Figure 1). The observed structures are consistent with the multicomponent membrane, and in particular with the hybrid membrane morphologies described in the literature. ${ }^{23-25}$ The simplest morphology corresponds to a homogeneous mixture of the copolymer and the lipid within the membrane. The signals of PE-Rhodamine and PDMS-NBD are, in this case, visible throughout the vesicle. This morphology is called "homogeneous membrane" (Figure 1- A and a). An inhomogeneous distribution of the probes within the membrane indicates the formation of copolymer or lipid-enriched domains as a result of phase separation (Figure 1- B and b). The spherical shape of the vesicle is maintained and, in most cases, only one domain per vesicle is visible. The size of the domains can vary, showing a slight difference in lipid fraction from one vesicle to another. Another morphology observed corresponds to the separation of the vesicles into two hemispheres, each incorporating a different probe. This shape is due to the phenomenon of budding of a domain outside the plane of the membrane (Figure 1- C and c). Vesicles marked only by one of the two probes have been also observed. These vesicles are enriched in either polymer or lipid, and result from the fission of hybrid membranes through the budding process (Figure 1- $\mathrm{C}$ and $\mathrm{d}$ ).
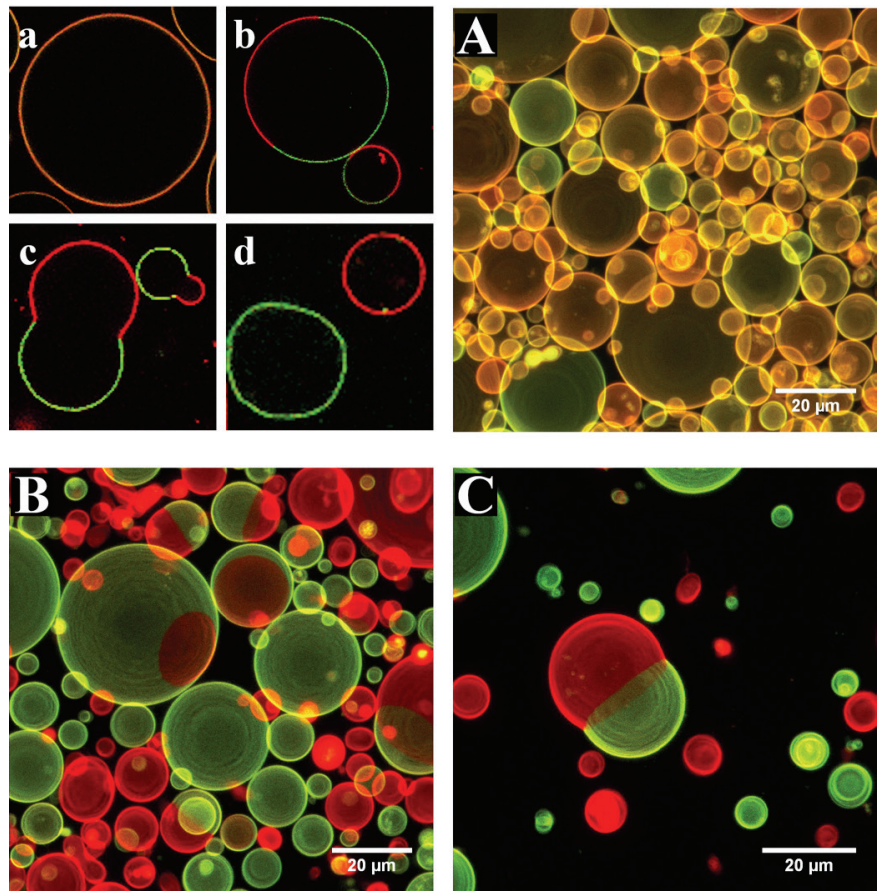

Figure 1 - A, B and C- 3D reconstruction based on confocal images taken along the zaxis illustrating the different structuration. a- Equatorial image of a GHUV presenting a homogeneous membrane (PDMS-NBD and rhodamine-PE perfectly overlapped). b- 
Equatorial image of a GHUV presenting domains while maintaining the spherical shape of the vesicle. c- Equatorial image of a GHUV presenting a budded part (growing of a second spherical structure from a preformed domain). $d$ - Equatorial image of a GUV composed exclusively of lipid resulting from the fission of a budded GHUV.

By counting the proportion of each structuration at different lipid content, we were able to draw the apparent phase diagram for the three hybrid systems (Figure 2).

In the case of the two shortest diblock copolymers $\left(\mathrm{Si}_{23} \mathrm{EO}_{13}\right.$ and $\mathrm{Si}_{27} \mathrm{EO}_{17}$ ), GHUVs are formed over the entire range of POPC fractions, as observed for their triblock analogs. ${ }^{23}$ Whatever the copolymer used, homogeneous membranes were formed at low POPC content. The POPC is dispersed in the continuous polymer phase until a limit fraction is reached. Above this limit ( $50 \mathrm{wt} . \%$ for $\mathrm{Si}_{23} \mathrm{EO}_{13}, 50 \mathrm{wt} . \%$ for $\mathrm{Si}_{27} \mathrm{EO}_{17}$ and 30 wt.\% for $\mathrm{Si}_{36} \mathrm{EO}_{23}$ ), the demixing of the system is favored. Evidence of phase separation can be found in systems composed of $\mathrm{Si}_{23} \mathrm{EO}_{13}$ and $\mathrm{Si}_{27} \mathrm{EO}_{17}$. The domains enriched in lipid or copolymer are both circular, illustrating the fluidity of the membrane. About $25 \%$ of the vesicles form buds, especially for $\mathrm{Si}_{23} \mathrm{EO}_{13}$ at about 60 wt.\% POPC. The buds can be formed either from lipid or polymer domains and are stable over two to four days. By further increasing the lipid fraction, GHUVs tend to fission. The fission fraction becomes predominant for $\mathrm{Si}_{23} \mathrm{EO}_{13}$ from 65 wt.\% POPC, and for $\mathrm{Si}_{27} \mathrm{EO}_{17}$ from 55 wt.\% POPC.

The behavior of $\mathrm{Si}_{36} \mathrm{EO}_{23}$ differs from other systems: homogeneous membranes were formed at low lipid fractions, however no GHUV could be observed above 40 wt.\% in POPC. Liposomes were visible, but they did not appear to be the result of GHUV fission as no intermediate step (formation of spherical domains and/or budding) could be observed. The polymer was sometimes present as aggregates, attached to the liposome membrane (see Figure S2 in ESI).

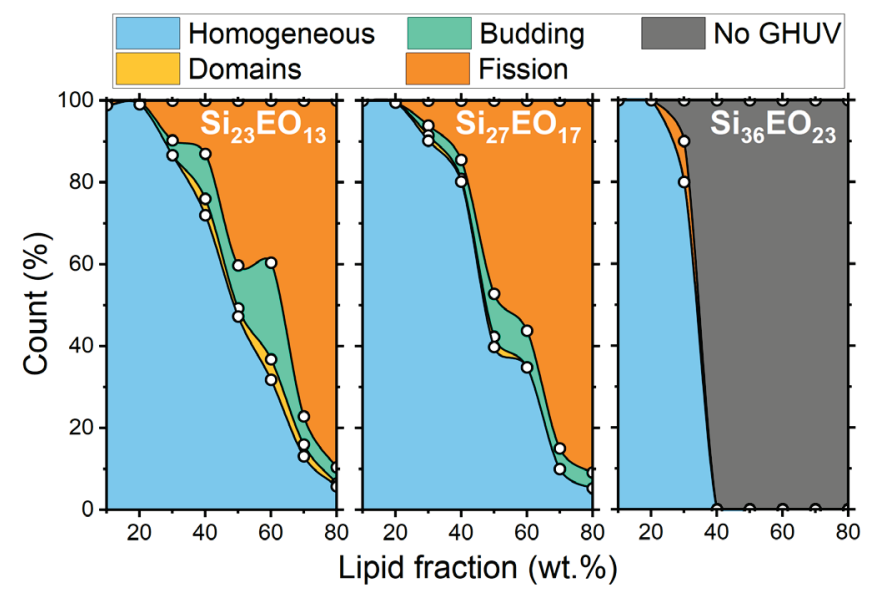

Figure 2 - Apparent phase diagrams of the GHUV formed from POPC and the diblock copolymers.

\section{Mechanical properties}

We studied the mechanical properties of the GHUV's membrane. The objective was to evaluate the effect of the membrane composition and to verify that hybrid vesicles do indeed have an interest in terms of mechanical strength compared to liposomes. Hybrid systems developed from triblock copolymers have shown lower toughness than those of liposomes. ${ }^{29}$ From literature it has been observed that vesicles obtained from PBD- $b$-PEO diblock copolymers show tenacities much higher than those of POPC but also higher than those obtained from PEO- $b$-PDMS- $b$-PEO triblock copolymers $^{25}$. As vesicles obtained from PDMS- $b$-PEO copolymers present very high tenacities, ${ }^{32}$ even higher than vesicle from PBD- $b$-PEO reported in literature, advantageous mechanical properties for GHUVs obtained from these diblock PDMS- $b$-PEO copolymers are expected. We evaluated membrane properties on vesicles with homogeneous membrane structuration using the micropipette suction method.

The evolution of the membrane structuration of each system was first checked under tension. Indeed, in one of our previous study, it has been found that for hybrid vesicles composed of POPC and triblock copolymers, micro-domains can appear under stress when the membrane composition is close to the limit where micro-domains spontaneously appear. These micro-domains result from the coalescence of nano-domains which were revealed by FLIM FRET. ${ }^{23}$ For all the systems studied, the membranes remain homogeneous under tension (Figure 3).
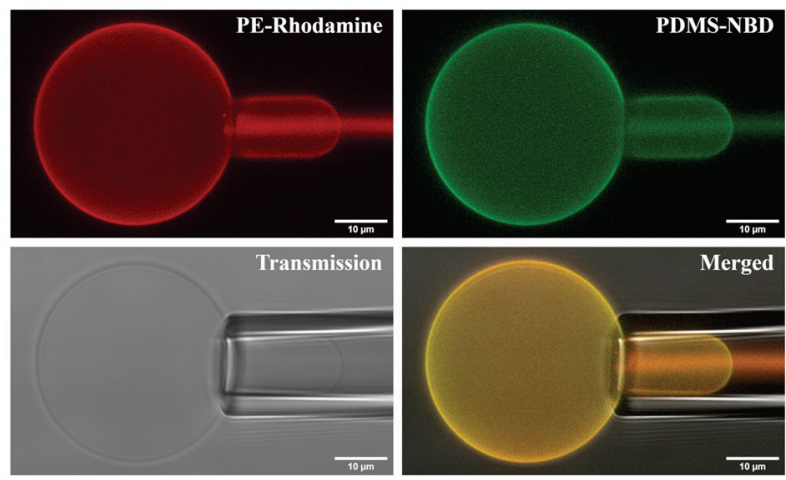

Figure 3 - Confocal images of micropipettes experiment in the different observation modes: PE Rhodamine channel, PDMS NBD channel, PE-Rhodamine and PDMS NBD channel merged, and transmission.

The mechanical properties were then determined in the absence of any probe, in order to measure the intrinsic properties of the membrane. The study carried out here is limited to POPC fractions up to $20 \mathrm{wt} . \%$. The mechanical properties of the homogeneous membranes were measured by micropipette aspiration until rupture was reached. Pure liposomes were also studied. The curves obtained (Figure S3) were fitted using Equation 6 to determine the area expansion 
modulus. The lysis strain $\left(\gamma_{c}\right)$ and stress $\left(\sigma_{c}\right)$ were determined from the abscissa and ordinate of the last point of the curves, respectively. Membrane toughness was obtained by calculating the area under the curves. The mean curves (Figure 4) were plotted using the mean area expansion modulus and the mean lysis strain determined using Equation 6 (Table S1).

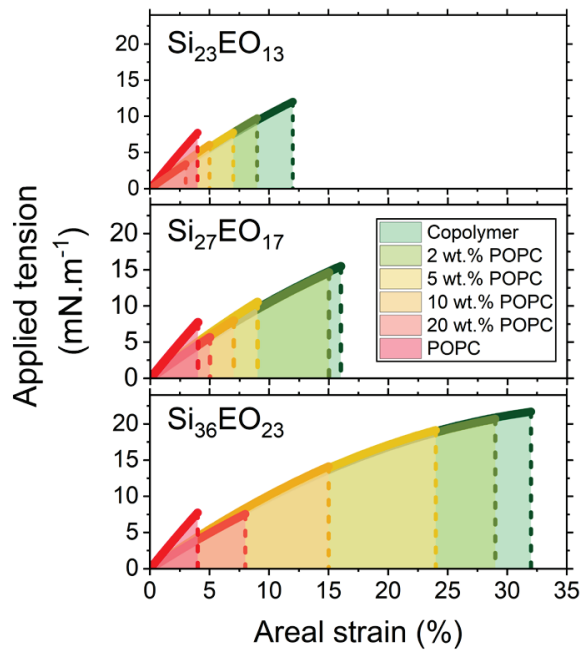

Figure 4 - Average stress strain curves of the GHUVs prepared from $\mathrm{Si}_{23} \mathrm{EO}_{13} / \mathrm{POPC}$ (top panel), $\mathrm{Si}_{27} \mathrm{EO}_{17} / \mathrm{POPC}$ (middle panel) and $\mathrm{Si}_{36} \mathrm{EO}_{23} / \mathrm{POPC}$ (bottom panel). Colored areas under the curves represent the average toughness.

The area expansion moduli of the GHUVs is almost constant whatever the lipid composition, between 100 and $130 \mathrm{mN} . \mathrm{m}^{-1}$. However, lysis strain and stress decrease progressively with the lipid fraction and increase with the molecular weight of the hydrophobic block (Figure 5). The lysis strain remains higher than that of POPC, except for GHUVs composed of the lower molar mass copolymer $\left(\mathrm{Si}_{23} \mathrm{EO}_{13}\right)$ with 20 wt.\% POPC. Concerning the lysis stress, the systems show lower values than POPC above $5 w$ t.\% for GHUVs composed of $\mathrm{Si}_{23} \mathrm{EO}_{13}, 10$ wt.\% for GHUVs composed of $\mathrm{Si}_{27} \mathrm{EO}_{17}$ and 20 wt.\% for GHUVs composed of $\mathrm{Si}_{36} \mathrm{EO}_{23}$.

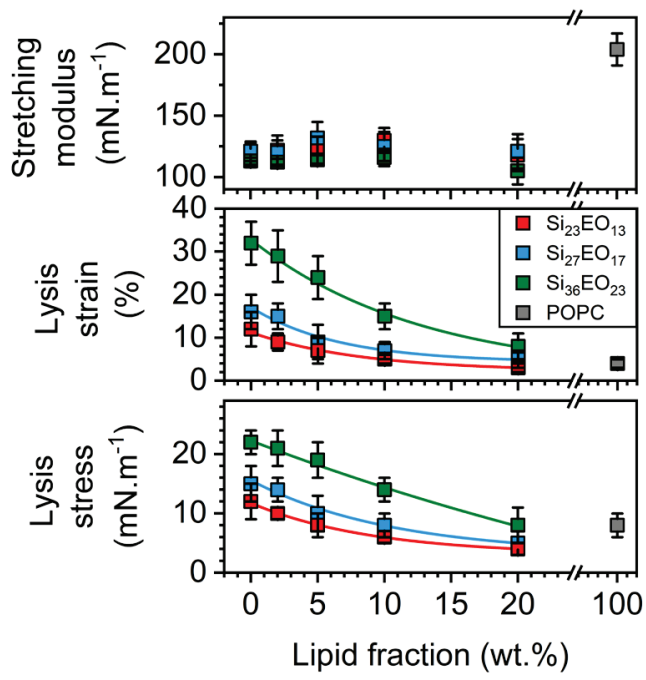

Author manuscript version
Figure 5 - Mechanical properties determined from the stress strain curves: Stretching modulus (top panel), lysis strain (middle panel) and lysis stress (bottom panel).

To compare the mechanical resistance of GHUVs, as previously stated, we measured the toughness of the hybrid membranes by calculating the area under curve. A decrease in toughness with the POPC fraction is observed (Figure 6). GHUVs composed of $\mathrm{Si}_{23} \mathrm{EO}_{13}$ have the lowest tenacities and therefore the most fragile membranes. The tenacity of this system remains higher than that of POPC up to $10 \mathrm{wt} \%$ in POPC. GHUVs composed of $\mathrm{Si}_{27} \mathrm{EO}_{17}$ have a higher tenacity than lipid membranes up to a fraction of $20 \mathrm{wt} . \%$ in POPC. Finally, GHUVs made of $\mathrm{Si}_{36} \mathrm{EO}_{23}$ have the most resistant membranes, with a tenacity always higher than POPC whatever the fraction of POPC in the studied range. For comparison, the triblock $\mathrm{PEO}_{12}-b-\mathrm{PDMS}_{43}-b-\mathrm{PEO}_{12}$ forms GHUVs with a membrane toughness lower than POPC even at low lipid fraction (5 wt.\% POPC). ${ }^{29}$

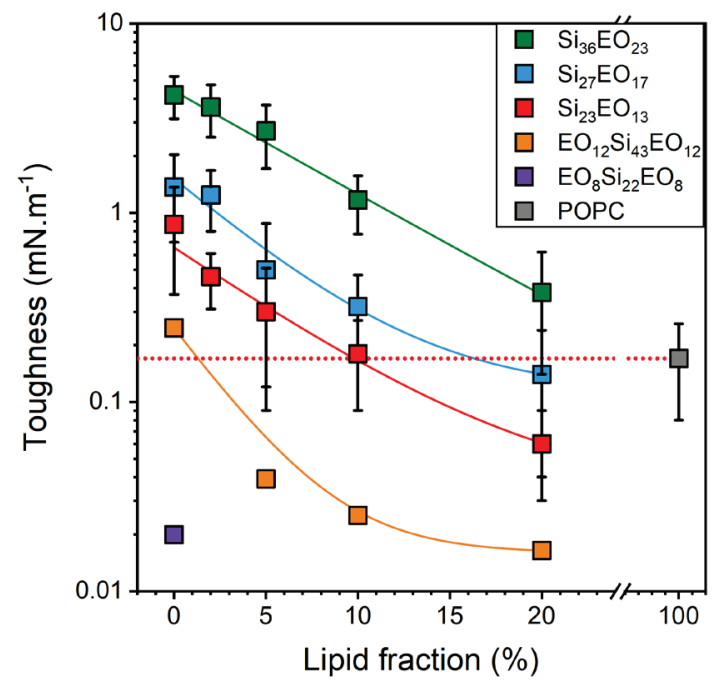

Figure 6 - Toughness of the GHUV composed of diblock and triblock copolymers. The dashed red line is a guide for the eye to show the typical toughness of a liposome.

\section{Discussion}

The membrane structuration of the GHUVs has been studied over the entire range of POPC compositions using PERhodamine as the lipid marker and PDMS-NBD as the polymer marker. The observed structuration are in agreement with those reported for different hybrid systems: a homogeneous mixture for low lipid fractions is obtained, followed by phase separation beyond a certain limit fraction, up to budding and fission phenomena as the lipid fraction increases (Figure 2). ${ }^{23 \text {, }}$ ${ }^{30}$ The structures obtained do not differ from those obtained for GHUVs composed of PEO- $b$-PDMS- $b$-PEO triblock copolymers. The diblock copolymers used in this study lead to the formation of membrane with similar thicknesses than those investigated with these triblock copolymers. Therefore, it appears that for identical chemical nature, the architecture of the copolymer used has no significant effect on the membrane structuration of the GHUVs. Indeed, the phase 
separation phenomenon for GHUVs, formed from diblock copolymers, is comparable to that observed with triblock copolymers. The boundary fractions follow more or less the same evolution with the hydrophobic mismatch whatever the architecture of the copolymer. The difference in line tension at the lipid polymer boundaries associated with the different conformations of diblock and triblock copolymers in the membrane is probably not high enough to create a difference in the phase separation phenomenon.

In the same manner as for the triblock copolymers, the hydrophobic length mismatch between the polymer and the lipid strongly influences the structuration of the membrane (homogeneous mixing or phase separation) as well as the stability of the domains and buds once the phase separation achieved.

For a small hydrophobic length difference (e.g. hybrids composed of $\mathrm{Si}_{23} \mathrm{EO}_{13}$ ), the polymer/lipid interface is stable enough to produce heterogeneous membranes. Thus, a significant number of GHUVs will present domains, as well as buds (up to $25 \%$ buds for $60 \mathrm{wt} . \%$ in POPC). An intermediate hydrophobic length difference (e.g. hybrids composed of $\mathrm{Si}_{27} \mathrm{EO}_{17}$ ) will produce less GHUV showing domains and buds. This originates probably from a higher line tension at the polymer/lipid interfaces, leading more rapidly to the formation of polymersomes and liposomes by budding and fission phenomenon. This budding phenomenon allows a decrease of the interfacial energy by reducing the interfacial length when line tension is too high $^{36,37}$ and can ultimately evolve towards fission. For example, $50 \%$ fission is obtained for $55 \mathrm{wt} . \%$ in POPC with $\mathrm{Si}_{27} \mathrm{EO}_{17}$, whereas the lipid fraction has to be increased to $65 \mathrm{wt} . \%$ to obtain the same amount of fission with $\mathrm{Si}_{23} \mathrm{EO}_{13}$.

Finally, a high hydrophobic length difference (e.g. hybrids composed of $\mathrm{Si}_{36} \mathrm{EO}_{23}$ ) will prevent the formation of GHUV with domains or buds. The incompatibility between the polymer and lipid phase may be so high that the polymer tolerates a very low amount of lipid, and almost complete phase separation occurs as soon as the film is formed.

Micropipette suction experiments have been used to determine the mechanical properties of GHUVs with a homogeneous membrane at the micrometer scale and assess the influence of the lipid fraction and length of the copolymer hydrophobic block. The high hydrophobic length difference for $\mathrm{Si}_{36} \mathrm{EO}_{23}$ does not appear to cause membrane embrittlement. GHUVs composed of diblock copolymers have mechanical properties far superior to what could be obtained for triblock systems. The origin of the brittleness of hybrid systems formulated from triblock copolymers and the outstanding toughness of those obtained from diblock copolymers of the same chemical nature remains to be determined. It is possible that differences in chain conformation in the membrane (between triblock and diblock copolymers) may play a role. Pure bilayer conformation is expected for a diblock copolymer whereas a mixture of extended and loop conformation is expected for a triblock copolymer. ${ }^{38,39}$ The GHUV membrane from diblock copolymers did not show any sign of nanodomains segregation and merging into micrometric domains, as described for triblock copolymers based GHUV. ${ }^{23}$ The existence of lipid nanodomains is therefore unlikely.

The values of stretching moduli are very close to the stretching moduli obtained for polymersomes and do not vary significantly with the lipid fraction (Figure 6 ) in contrast to what has been observed for hybrid systems based on PEO- $b$ PDMS- $b$-PEO/POPC ${ }^{29}$ and PBD- $b$-PEO/POPC ${ }^{25}$. This result is surprising in that a modulation of the membrane composition should lead to a change in the interfacial tension between hydrophilic and hydrophobic parts and thus a change in the stretching modulus. ${ }^{40,41}$ The latter therefore seems, not to be influenced by the presence of the lipid in the membrane.

\section{Conclusions}

In this work we reported a systematic study of the formation of GHUV from the self-assembly of PDMS- $b$-PEO diblock copolymers and POPC, and their mechanical properties. A comparison with a previous study realized with triblock PEO- $b$ PDMS- $b$-PEO copolymers leading to the formation of vesicle with membrane thickness similar to those of diblock copolymer studied here has been performed. Globally the evolution of membrane structuration follows the same tendency with lipid composition whatever the architecture of the copolymer. Therefore, it seems that the different conformation of polymer chains (extended and loop for triblock, bilayer for diblock) do not play a major role in the membrane structuration. However, a huge difference has been evidenced in term of membrane toughness. GHUV obtained with PDMS- $b$-PEO diblock copolymer show outstanding toughness especially for high molar masses even at relatively high fraction of POPC (15-20 wt.\%) whereas it has been shown previously that association of PEO- $b$-PDMS- $b$-PEO triblock copolymer with POPC led to the formation of GHUV that could be even more fragile than pure liposomes. Quantitative measurements of line tension at the lipid polymer boundaries could help to improve the qualitative interpretation of such differences and are planned in a further study. We think that this architecture effect could be extrapolated on copolymers of different chemical nature. Indeed, for instance, diblock PBD$b$-PEO copolymers which are one of the most investigated block copolymers to develop hybrid vesicles presents, at low lipid fraction, similar tendencies in term of membrane structuration and mechanical properties. ${ }^{25}$ Such hypothesis should be confirmed in further studies.

\section{Conflicts of interest}

There are no conflicts to declare.

\section{Acknowledgements}

M. Fauquignon gratefully acknowledges Scientific Department of University of Bordeaux for PhD fellowship. 


\section{Notes and references}

1. A. Krywko-Cendrowska, S. di Leone, M. Bina, S. YorulmazAvsar, C. G. Palivan and W. Meier, Polymers, 2020, 12, 1003.

2. M. Schulz and W. H. Binder, Macromolecular Rapid Communications, 2015, 36, 2031-2041.

3. J. F. Le Meins, C. Schatz, S. Lecommandoux and O. Sandre, Mater. Today, 2013, 16, 397-402.

4. T. P. T. Dao, K. Ferji, F. Fernandes, M. Prieto, S. Lecommandoux, E. Ibarboure, O. Sandre and J.-F. Le Meins, in The Giant Vesicle Book, eds. C. M. Marques and D. Dimova, CRC Press, 2020, ch. 27.

5. C. Kleineberg, C. Wolfer, A. Abbasnia, D. Pischel, C. Bednarz, I. Ivanov, T. Heitkamp, M. Borsch, K. Sundmacher and T. Vidakovic-Koch, Chembiochem, 2020.

6. T. Nishimura, S. Hirose, Y. Sasaki and K. Akiyoshi, J Am Chem Soc, 2020, 142, 154-161.

7. S. Di Leone, S. Y. Avsar, A. Belluati, R. Wehr, C. G. Palivan and W. Meier, J Phys Chem B, 2020, 124, 4454-4465.

8. Y. Zhang, N. Gal, F. Itel, I. N. Westensee, E. Brodszkij, D. Mayer, S. Stenger, M. Castellote-Borrell, T. Boesen, S. R. Tabaei, F. Höök and B. Städler, Nanoscale, 2019, 11, 11530-11541.

9. P. A. Beales, S. Khan, S. P. Muench and L. J. C. Jeuken, Biochem. Soc. Trans., 2017, 45, 15-26.

10. W. F. Paxton, P. T. McAninch, K. E. Achyuthan, S. H. R. Shin and H. L. Monteith, Colloids Surf. B, 2017, 159, 268-276.

11. R. Seneviratne, S. Khan, E. Moscrop, M. Rappolt, S. P. Muench, L. J. C. Jeuken and P. A. Beales, Methods, 2018, 147 142-149.

12. S. Khan, M. Li, S. P. Muench, L. J. C. Jeuken and P. A. Beales, Chem. Commun., 2016, 52, 11020-11023.

13. S. Khan, J. McCabe, K. Hill and P. A. Beales, J Colloid Interface Sci, 2020, 562, 418-428.

14. M. Mumtaz Virk and E. Reimhult, Langmuir, 2018, 34, 395405.

15. N. Pippa, M. Merkouraki, S. Pispas and C. Demetzos, Int J Pharm, 2013, 450, 1-10.

16. S. Lim, H.-P. de Hoog, A. Parikh, M. Nallani and B. Liedberg, Polymers, 2013, 5, 1102-1114.

17. N. Pippa, D. Stellas, A. Skandalis, S. Pispas, C. Demetzos, M. Libera, A. Marcinkowski and B. Trzebicka, Eur J Pharm Biopharm, 2016, 107, 295-309.

18. K. Panneerselvam, M. E. Lynge, C. F. Riber, S. MenaHernando, A. A. Smith, K. N. Goldie, A. N. Zelikin and B. Stadler, Biomicrofluidics, 2015, 9, 052610.

19. Z. Cheng, D. R. Elias, N. P. Kamat, E. Johnston, A. A Poloukhtine, V. V. Popik, D. A. Hammer and A. Tsourkas, Bioconjugate Chem., 2011, 22, 2021-2029.

20. M. Schulz, S. Werner, K. Bacia and W. H. Binder, Angew Chem Int Ed Engl, 2013, 52, 1829-1833.

21. S. K. Lim, A. S. W. Wong, H.-P. M. de Hoog, P. Rangamani, A. N. Parikh, M. Nallani, S. Sandin and B. Liedberg, Soft Matter, 2017, 13, 1107-1115.

22. S.-W. Hu, C.-Y. Huang, H.-K. Tsao and Y.-J. Sheng, Physical Review E, 2019, 99, 012403.
23. T. P. T. Dao, F. Fernandes, E. Ibarboure, K. Ferji, M. Prieto, O. Sandre and J.-F. Le Meins, Soft Matter, 2017, 13, 627-637.

24. J. Nam, T. K. Vanderlick and P. A. Beales, Soft Matter, 2012, 8, 7982-7988.

25. J. Nam, P. A. Beales and T. K. Vanderlick, Langmuir, 2011, 27, 1-6.

26. M. Chemin, P. M. Brun, S. Lecommandoux, O. Sandre and J. F. Le Meins, Soft Matter, 2012, 8, 2867-2874.

27. W. Shen, J. Hu and X. Hu, Chem. Phys. Lett., 2014, 600, 5661.

28. M. Kang, B. Lee and C. Leal, Chemistry of Materials, 2017, 29 , 9120-9132.

29. T. P. T. Dao, F. Fernandes, M. Fauquignon, E. Ibarboure, M. Prieto and J. F. Le Meins, Soft Matter, 2018, 14, 6476-6484.

30. D. Chen and M. M. Santore, Soft Matter, 2015, 11, 26172626.

31. J. Y. Kang, I. Choi, M. Seo, J. Y. Lee, S. Hong, G. Gong, S. S. Shin, Y. Lee and J. W. Kim, J Colloid Interface Sci, 2020, 561, 318326.

32. M. Fauquignon, E. Ibarboure, S. Carlotti, A. Brûlet, M. Schmutz and J.-F. Le Meins, Polymers, 2019, 11, 2013.

33. T. P. T. Dao, A. Brûlet, F. Fernandes, M. Er-Rafik, K. Ferji, R. Schweins, J. P. Chapel, A. Fedorov, M. Schmutz, M. Prieto, O. Sandre and J. F. Le Meins, Langmuir, 2017, 33, 1705-1715.

34. M. I. Angelova and D. S. Dimitrov, Faraday Discuss. Chem. Soc., 1986, 81, 303-311.

35. E. Ibarboure, M. Fauquignon and J.-F. Le Meins, JoVE, 2020, e60199.

36. J. Wolff, S. Komura and D. Andelman, Phys Rev E Stat Nonlin Soft Matter Phys, 2015, 91, 012708.

37. M. Schulz, A. Olubummo, K. Bacia and W. H. Binder, Soft Matter, 2014, 10, 831-839.

38. F. Itel, M. Chami, A. Najer, S. Loercher, D. Wu, I. A. Dinu and W. Meier, Macromolecules, 2014, 47, 7588-7596.

39. H.-C. Tsai, Y.-L. Yang, Y.-J. Sheng and H.-K. Tsao, Polymers, 2020, 12, 639.

40. B. M. Discher, Y. Y. Won, D. S. Ege, J. C. M. Lee, F. S. Bates, D. E. Discher and D. A. Hammer, Science, 1999, 284, 1143-1146.

41. H. Bermudez, A. K. Brannan, D. A. Hammer, F. S. Bates and D. E. Discher, Macromolecules, 2002, 35, 8203-8208. 


\title{
Membrane reinforcement in Giant Hybrid Polymer Lipid Vesicle achieved by controlling the polymer architecture
}

\author{
By Martin Fauquignon, ${ }^{a}$ Emmanuel Ibarboure a Jean-François Le Meins*
}

a. Université de Bordeaux, CNRS, Bordeaux INP, LCPO, UMR 5629, F-33600, Pessac, France.

Brief recall of the synthesis procedure of the copolymers, published in 1,2

\section{Synthesis of triblock copolymers ${ }^{2}$}

The amphiphilic triblock copolymers $\mathrm{PEO}_{8}-b-\mathrm{PDMS}_{22}-b-\mathrm{PEO}_{8}$ and $\mathrm{PEO}_{12}-b-\mathrm{PDMS}_{43}-b-\mathrm{PEO}_{12}$ were synthesized via coupling of two hydrophilic PEO blocks onto a hydrophobic PDMS block. Hydrophobic blocks were the $\alpha, \omega$-bisaminopropyl-terminated poly(dimethylsiloxane) ( $\left.\mathrm{NH}_{2}-\mathrm{PDMS}-\mathrm{NH}_{2}\right)$ with a number average molecular weight of 1500 and 3000 g.mol ${ }^{-1}$ according to the provider's data (ABCR, Germany) and as also verified by ${ }^{1} \mathrm{H} N M R$, defining 22 , and 43 units for PDMS chain respectively. Succinimidyl((N-methyl)-ethyleneglycol) ester (509 and $685.7 \mathrm{~g} / \mathrm{mol}$ ) or (PEO-NHS) corresponding to 8 and 12 units of PEO chain) were used as hydrophilic blocks, purchased from Thermo Fisher Scientific Inc. The coupling reactions were carried out under an inert gas in dichloromethane with the presence of DIPEA during $24 \mathrm{~h}$ at room temperature. The obtained products were then purified by dialysis using a membrane with MWCO of 50kDa. The copolymer was characterized by ${ }^{1} \mathrm{H} \mathrm{NMR}$ in $\mathrm{CDCl}_{3}$ and Size Exclusion Chromatography in THF.

\section{Synthesis of diblock copolymers ${ }^{1}$}

All The diblock copolymers PDMS- $b$-PEO in this study were synthesized by ring-opening polymerization of a cyclic siloxane monomer $\left(D_{3}\right)$, functionalization of the PDMS chain and further coupling with alkyne end-functionalized poly(ethylene oxide) using Huisgen's coupling. For that, hexamethylcyclotrisiloxane, dried over $\mathrm{CaH}_{2}$ and dissolved in freshly distilled THF, was polymerized under argon using butyllithium in cyclohexane/hexane. The solution was stirred at room temperature for $2 \mathrm{~h}$ then the reaction was quenched by adding chloro-(3chloropropyl)dimethylsilane. After filtration, washing in methanol and further drying process the polymer was characterized by $1 \mathrm{H}$ NMR, SEC in toluene, and FT-IR. Then, the end-chain was functionalized with an azido group by addition of $\mathrm{NaN}_{3}$ into a solution of PDMS-Cl in dimethoxyethane and dimethylformamide (50/50 vol./vol.). After dilution in pentane and washing procedure with water, the solvent was evaporated and product was dried under vacuum before characterization by ${ }^{1} \mathrm{H}$ NMR, SEC in toluene and FT-IR.

A commercial methoxy terminated PEO dissolved in freshly distilled THF was reduced by $\mathrm{NaH}$ and functionalized by addition of a solution of propargyl bromide at $80 \mathrm{wt} . \%$ in toluene. After $24 \mathrm{~h}$, the mixture was concentrated, then diluted in dichloromethane and washed with water. After a drying, the final product was characterized by ${ }^{1} \mathrm{H}$ NMR and SEC in THF. 
Finally, azido functionalized PDMS was then coupled to alkyne functionalized PEO in toluene. PMDETA and $\mathrm{CuBr}$ were added and reaction was conducted at $80^{\circ} \mathrm{C}$ for $24 \mathrm{~h}$. The solvent was then evaporated and the product was solubilized in dichloromethane and purified on silica column using a 7 vol.\% mix of methanol with dichloromethane as eluent phase. The product was dried under vacuum overnight and the final product was characterized by ${ }^{1} \mathrm{H} N \mathrm{NMR}$ and SEC in THF.

\section{Influence of fluorescent probe on mechanical properties of GUV}

We have evaluated the potential influence of the fluorescent probes used for confocal imaging, on the mechanical properties of GUV.

For that we have studied the behaviour of giant polymersomes obtained from the selfassembly of $\mathrm{Si}_{27} \mathrm{EO}_{17}$, without fluorescent probe, with $2 \%$ of PMDS-NBD, and in a last case with $0.2 \%$ of PE Rhodamine. We have also studied mechanical properties of GHUV without probes, with $0.2 \%$ of PE rhodamine and with both probe $0.2 \%$ PEI rhodamine and $2 \%$ PDMS-b-NBD The profile of the membrane tension versus the area expansion is represented for the different systems in the Figure $\mathrm{S} 3$.
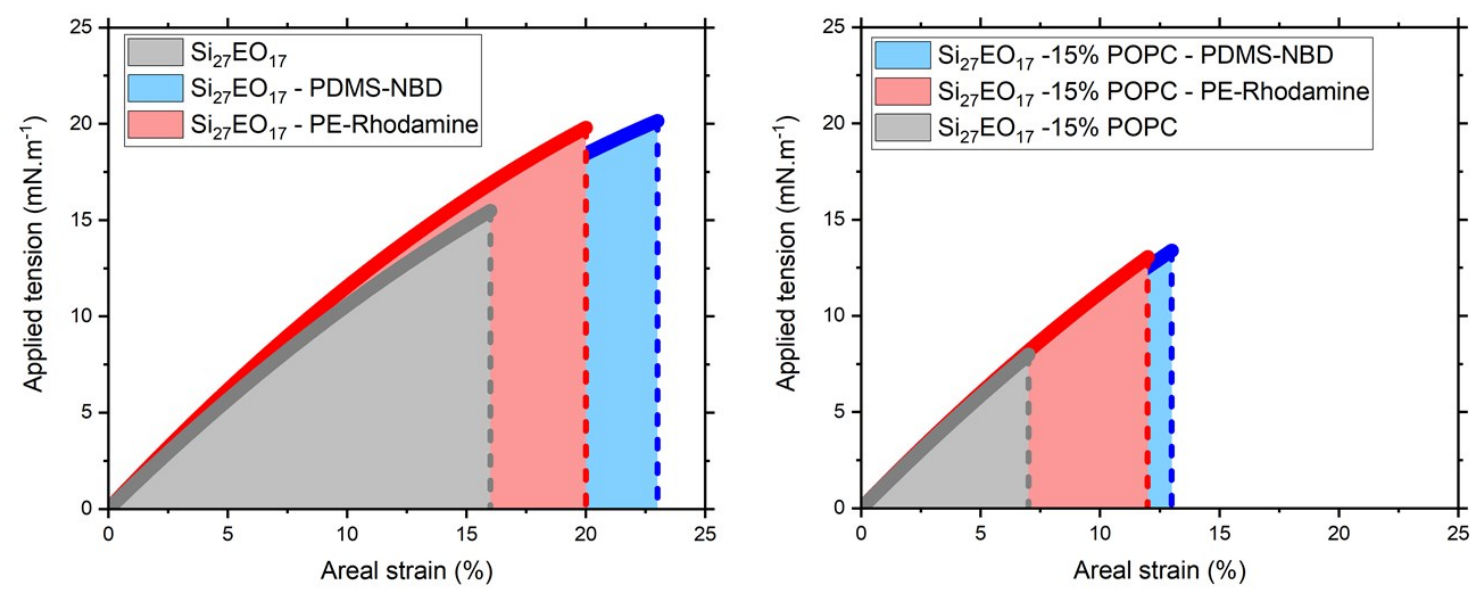

Figure S1 left: Membrane tension versus area expansion for the polymersomes with different probes. Right B Same thing for GHUV (15\% POPC) with different probes.

It is clear from the results obtained that the presence of probes in the membrane has an influence on the mechanical properties. If the stretching moduli are almost unchanged, the Lysis strain is modulated and can be higher or lower that the lysis strain of the pure polymersomes or GHUV. We do not have clear explanation of this experimental fact. All the mechanical properties of the GHUV in this article were analyzed without fluorescent probes. 
Appearance of polymer aggregation for the $\mathrm{Si}_{36} \mathrm{EO}_{23} / \mathrm{POPC}$ system
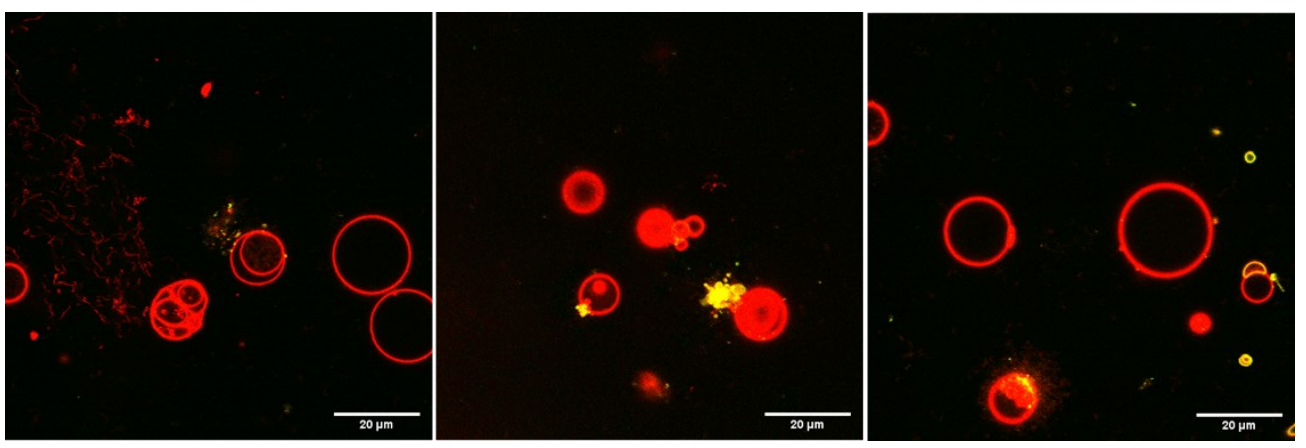

Figure $\mathbf{S 2}$ - Confocal images showing the aggregation of the $\mathrm{Si}_{36} \mathrm{EO}_{23}$ on membranes formed by POPC when the lipid fraction is above $40 \mathrm{wt} . \%$ in the initial mixture. 


\section{Mechanical properties measurements}
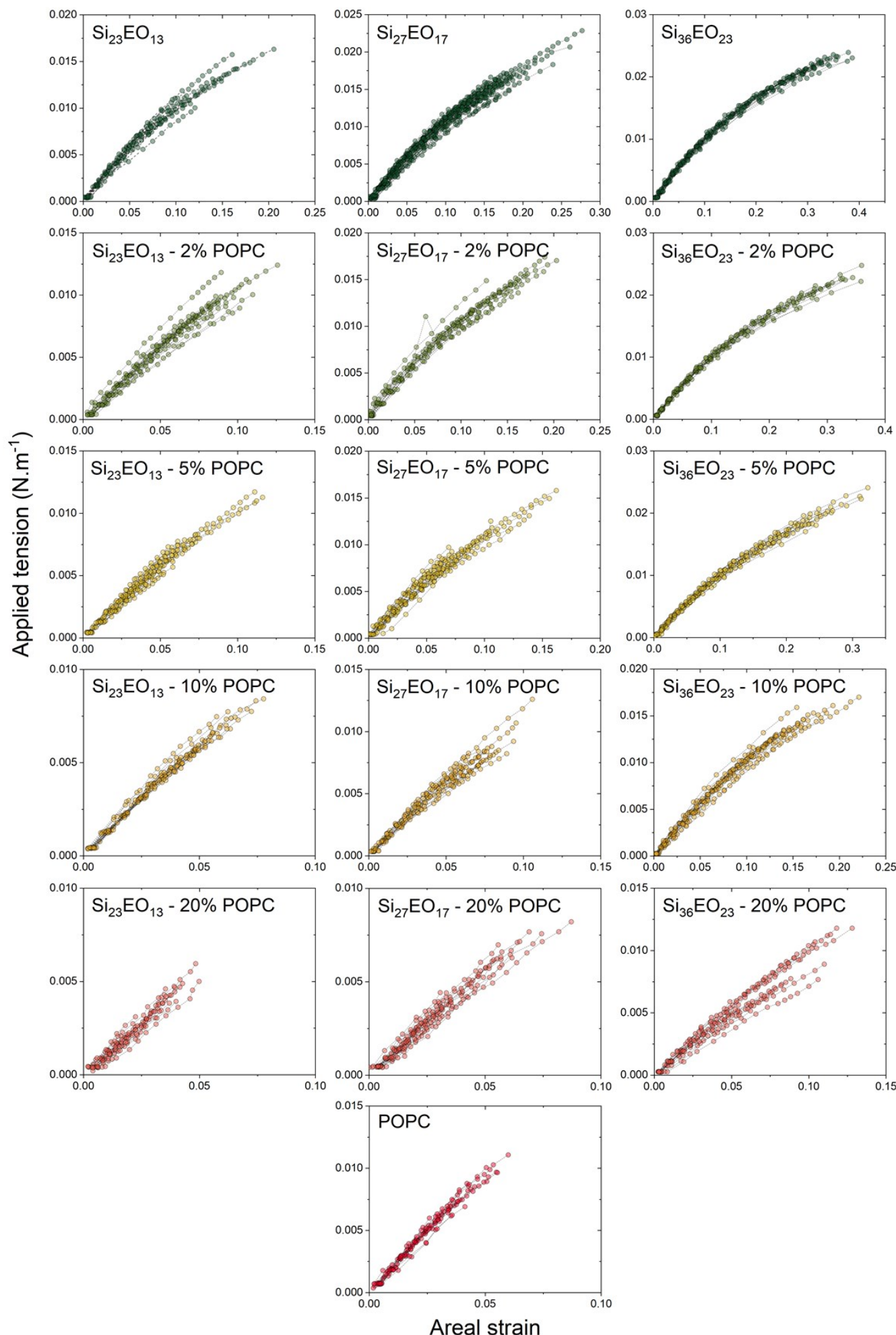

Figure S3 - Stress strain curves of GHUVs prepared from $\mathrm{Si}_{23} \mathrm{EO}_{13}, \mathrm{Si}_{27} \mathrm{EO}_{17}$ and $\mathrm{Si}_{36} \mathrm{EO}_{23}$ at different POPC fractions. 
Table S1: Mechanical properties of Hybrid vesicle's membranes

\begin{tabular}{|c|c|c|c|c|c|}
\hline & $\begin{array}{l}\text { POPC fraction } \\
\text { (wt.\%) }\end{array}$ & $\begin{array}{l}\text { Stretching modulus } \\
\qquad\left(\mathrm{mN} \cdot \mathrm{m}^{-1}\right)\end{array}$ & $\begin{array}{l}\text { Lysis strain } \\
\text { (\%) }\end{array}$ & $\begin{array}{l}\text { Lysis stress } \\
\left(\mathrm{mN} \cdot \mathrm{m}^{-1}\right)\end{array}$ & $\begin{array}{c}\text { Toughness } \\
\left(\mathrm{mN} \cdot \mathrm{m}^{-1}\right)\end{array}$ \\
\hline \multirow{5}{*}{$\mathrm{Si}_{23} \mathrm{EO}_{13}$} & 0 & $118 \pm 10$ & $12 \pm 4$ & $12 \pm 3$ & $0,87 \pm 0,50$ \\
\hline & 2 & $122 \pm 12$ & $9 \pm 2$ & $10 \pm 1$ & $0,46 \pm 0,15$ \\
\hline & 5 & $122 \pm 11$ & $7 \pm 3$ & $8 \pm 2$ & $0,30 \pm 0,21$ \\
\hline & 10 & $130 \pm 10$ & $5 \pm 1$ & $6 \pm 1$ & $0,18 \pm 0,09$ \\
\hline & 20 & $118 \pm 13$ & $3 \pm 1$ & $4 \pm 1$ & $0,06 \pm 0,03$ \\
\hline \multirow{5}{*}{$\mathrm{Si}_{27} \mathrm{EO}_{17}$} & 0 & $121 \pm 8$ & $16 \pm 4$ & $15 \pm 3$ & $1,37 \pm 0,67$ \\
\hline & 2 & $120 \pm 10$ & $15 \pm 3$ & $14 \pm 2$ & $1,24 \pm 0,44$ \\
\hline & 5 & $132 \pm 13$ & $9 \pm 4$ & $10 \pm 3$ & $0,50 \pm 0,38$ \\
\hline & 10 & $125 \pm 12$ & $7 \pm 2$ & $8 \pm 2$ & $0,32 \pm 0,15$ \\
\hline & 20 & $121 \pm 14$ & $5 \pm 2$ & $5 \pm 2$ & $0,14 \pm 0,10$ \\
\hline \multirow{5}{*}{$\mathrm{Si}_{36} \mathrm{EO}_{23}$} & 0 & $113 \pm 3$ & $32 \pm 5$ & $22 \pm 2$ & $4,20 \pm 1,06$ \\
\hline & 2 & $112 \pm 3$ & $29 \pm 6$ & $21 \pm 3$ & $3,63 \pm 1,12$ \\
\hline & 5 & $114 \pm 4$ & $24 \pm 5$ & $19 \pm 3$ & $2,71 \pm 1,00$ \\
\hline & 10 & $116 \pm 7$ & $15 \pm 3$ & $14 \pm 2$ & $1,17 \pm 0,40$ \\
\hline & 20 & $105 \pm 11$ & $8 \pm 3$ & $8 \pm 3$ & $0,38 \pm 0,24$ \\
\hline POPC & 100 & $204 \pm 13$ & $4 \pm 1$ & $8 \pm 2$ & $0,17 \pm 0,09$ \\
\hline
\end{tabular}

(1) Fauquignon, M.; Ibarboure, E.; Carlotti, S.; Brûlet, A.; Schmutz, M.; Le Meins, J.-F. Polymers 2019, 11, 2013.

(2) Dao, T. P. T.; Brûlet, A.; Fernandes, F.; Er-Rafik, M.; Ferji, K.; Schweins, R.; Chapel, J. P.; Fedorov, A.; Schmutz, M.; Prieto, M.; Sandre, O.; Le Meins, J. F. Langmuir 2017, 33, 1705. 